\title{
FEATURES OF TEXTURE FORMATION IN POLYMORPHIC METALS BEING ELECTRODEPOSITED
}

\author{
Oleg B. Girin ${ }^{1) *}$, Volodymyr I. Ovcharenko ${ }^{1)}$, Dmytro G. Korolyanchuk ${ }^{1)}$ \\ 1) Ukrainian State University of Chemical Technology, Materials Science Department, Dnipro, \\ Ukraine
}

Received: 30.05.2019

Accepted: 02.12.2019

*Corresponding author: e-mail: girin@ua.fm, Materials Science Department, Ukrainian State

University of Chemical Technology, 8 Gagarin Ave, Dnipro 49005, Ukraine

\begin{abstract}
The aim of this work was further experimental verification of the existence of the phenomenon of electrochemical phase formation in metals and alloys via a supercooled liquid state stage. According to proposed idea the slowing down of the process of polymorphic transformation in a metal being electrodeposited should be accompanied by an intensive formation of the texture of the metastable modification and suppression of the texture development of the stable modification. The results of the texture analysis of electrodeposited cobalt as a model metal showed that under slowing down the polymorphic transformation process in a metal being electrodeposited, the texture formation is intensified in the metastable modification, but suppressed in the stable modification. The finding is another proof of the existence of the phenomenon of electrochemical phase formation in metals and alloys through a supercooled liquid state stage.
\end{abstract}

Keywords: electrochemical phase formation, electrodeposited cobalt, polymorphic transformation, texture

\section{Introduction}

The search for new methods and options for obtaining electrodeposited metals and alloys in the form of electrochemical coatings with improved physical-mechanical or chemical properties is hardly possible without information about their structure, texture, substructure, surface morphology and structural defects. Control of these structural characteristics that affect the properties of the resulting electrodeposited coatings is based on the concepts of the electrochemical phase formation of metals and alloys.

A previously unknown phenomenon of electrochemical phase formation in metals and alloys through a supercooled liquid state stage has been found relatively recently $[1,2]$. The phenomenon occurs during the electrochemical deposition of metals or alloys in an aqueous medium onto a solid cathode, when a deeply supercooled metal liquid evolves in the form of multitude liquid atomic clusters that appear in an avalanche-like manner at various places near the cathode or the growing deposit, and then solidifies ultra-rapidly at the deposition temperature.

The discovered phenomenon is due to an extremely rapid, burst-like liberation of the metal or alloy, resulting from a chain reaction of electrochemical formation of atoms and transition of the atomic clusters or their associations from a liquid to a more stable solid state [1].

Later, numerous works ([3-6] are the most recent) were published, in which the occurrence of this phenomenon was experimentally proved. 
The proposed concept is fundamentally different from the existing ones, according to which, the phase formation in electrodeposited metal or alloy occurs by embedding ions from an aqueous solution, or atoms formed on their surfaces, into their crystal lattice [7-11]. These concepts have received theoretical development in recent publications [12-15] on the electrochemical phase formation in metals and alloys.

Since the proposed concept of electrochemical phase formation in metals and alloys through a supercooled liquid state stage [1-6] has not yet received any objections or refutations, the authors continue to develop it with the introduction and implementation of new hypotheses and ideas.

\section{Principal Hypothesis}

It is known that in the process of solidification from the liquid state, the metallurgical polymorphic metals go through metastable phase states and crystallize as a modification that is stable under given thermodynamic conditions [16, 17]. If the phase transformation of a metastable modification into a stable one is slowed down or partially suppressed, a dual-phase state of the metal will eventually be formed. In the case of complete prevention of this phase transition, the solidified metal will stay in the metastable phase state.

The basic hypothesis of the experiments performed was that, in accordance with the phenomenon under discussion, the crystallization of a polymorphic metal being electrodeposited from the supercooled liquid state should be viewed as its sequential going through metastable modifications and, at the last stage of phase formation, emerging a modification that is stable under given deposition conditions.

\section{Idea of Experiment}

If polymorphic metals, being electrodeposited, indeed crystallize from the supercooled metallic liquid formed in the micro volumes near the surface of a deposit, then one can expect quite predictable features of the oriented grain growth in metastable and stable modifications in case the process of polymorphic transformation slows down during electrochemical phase formation. Specifically, in this case, the texturing of metastable modification of a polymorphic metal should intensify, while the texture development of a stable modification should be hindered.

The above texturing features in the metastable and stable modifications in the electrodeposits of polymorphic metals will testify to the phenomenon of electrochemical phase formation in metals and alloys through a supercooled liquid state stage.

\section{The Aim and Objectives of the Study}

The aim of this study was to further experimentally verify the existence of the phenomenon under discussion by investigating the features of texture formation in polymorphic metals. To achieve the object, the following tasks were set:

- qualitatively and quantitatively assess the crystallographic texture of metastable and stable modifications of a model polymorphic metal;

- investigate the change in the degree of texture perfection in each modification of the polymorphic metal under aggravating the conditions that slow down the phase transformation of the metastable modification into a stable one;

- compare the obtained dependences with the predicted changes in the texture of various modifications of the polymorphic metal in the case of slowing down the process of polymorphic transformation during the electrochemical phase formation; 
- perform additional verification experiments to make sure that the obtained results were correctly interpreted.

\section{Materials and Methods}

\subsection{Obtaining electrodeposited polymorphic metals}

As a model polymorphic metal, cobalt was used that exists in two modifications: a metastable $\beta$ modification with a face-centered cubic lattice ( $\beta$-Co), and a stable $\alpha$-modification with a hexagonal close-packed lattice ( $\alpha$-Co). Cobalt coatings $20 \mu \mathrm{m}$ thick were obtained by the method of electrodeposition at a temperature of $25^{\circ} \mathrm{C}$ in a solution of the composition $(\mathrm{g} / \mathrm{l})$ : $\mathrm{CoSO}_{4} \cdot 7 \mathrm{H}_{2} \mathrm{O}$ $-300, \mathrm{H}_{3} \mathrm{BO}_{3}-40, \mathrm{NaCl}-20$. The current density was changed over the interval from 0.5 to $10.0 \mathrm{~A} / \mathrm{dm}^{2}$, with the conventional adoption of 0.5-1.0 as mild, 1.5-6.5 as average and 7.0$10.0 \mathrm{~A} / \mathrm{dm}^{2}$ as hard electrodeposition conditions.

With an increase in the current density, the rate of electrochemical crystallization of cobalt increases [18], which in the case of an insignificant change in the current yield of cobalt leads to an increase in the amount of the metastable $\beta$-Co phase. Given the $\beta$-Co $\rightarrow \alpha$-Co polymorphic transformation in cobalt through the shear mechanism, an increase in the amount of the metastable $\beta$-Co modification will contribute to slowing down the process of polymorphic transformation.

\subsection{Study of the textural and structural characteristics}

The crystallographic texture of the electrodeposited cobalt was investigated using a modernized $\mathrm{X}$-ray diffractometer DRON-2 (Russia) in $\mathrm{Cr}-\mathrm{K}_{\alpha}$ radiation. Preliminary experiments revealed that the cobalt coatings under study featured both the stable $\alpha$-Co phase and metastable $\beta$-Co phase. Given the two-phase structure of cobalt coatings, the preferred grain orientation in each phase was determined by a combination of direct pole figures. The technique involved recording texturerelevant curves of interference by $\{111\}$ for $\beta-\mathrm{Co}$ and $\{10.1\}$ for $\alpha$-Co, which were then used to plot (111) and (10.1) direct pole figures for $\beta$ - and $\alpha$-modifications of cobalt, respectively. For the quantitative estimation of the degree of texture perfection of two-phase cobalt coatings, the average scattering angle of the texture in each modification was used.

The surface morphology of the coatings was studied using a scanning electron microscope REM106I (Ukraine), operating in the mode of image formation by secondary electrons.

\section{Results}

As a result of the experiments performed, it was established that in all the modes of obtaining the coatings, both modifications of electrodeposited cobalt are textured. Moreover, a comparative analysis of texture-relevant curves of interferences by $\{111\}$ for $\beta$-Co (Fig. 1, a, c, e) and interferences by $\{10.1\}$ for $\alpha$-Co (Fig. 1, b, d, f) shows that the texture axes do not change with an increase in the rate of cobalt coating deposition; it is only the degree of perfection of the preferred grains orientation that undergoes changes in each phase.

The analysis of direct pole figures (111) for $\beta$-modification (Fig. 2, a) and (10.1) for $\alpha$ modification (Fig. 2, b) of electrodeposited cobalt showed that its texture is described by two axial orientations with [110] axis for $\beta$-Co and [11.0] for $\alpha$-Co, perpendicular to the sample surface, and by a random component.

Indeed, the presence of the textural maximum of the axial orientation of the grains with the [110] axis at an angle of $35.3^{\circ}$ in pole figure (111) for the $\beta$-modification of a two-phase cobalt sample (Fig. 2, a) in combination with the texture maximum of the axial orientation of the grains with the 
[11.0] axis at an angle of $40.1^{\circ}$ in (10.1) pole figure for the $\alpha$-modification of the same sample (Fig. 2, b) allowed this conclusion. It should be noted that in the hexagonal close-packed system, where $\alpha$-Co belongs, the [11.0] crystallographic direction and the (11.0) crystallographic plane are mutually perpendicular.

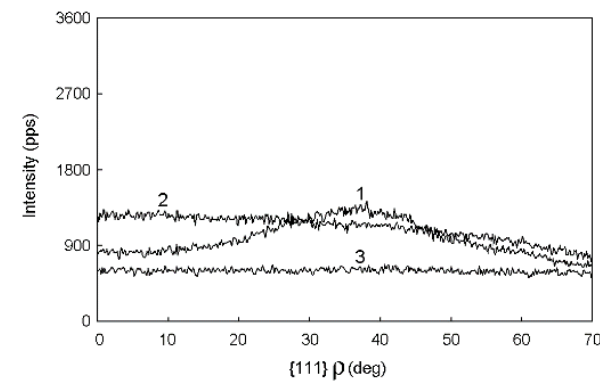

(a)

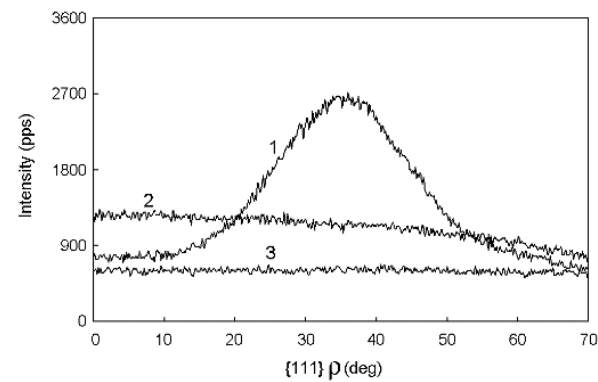

(c)

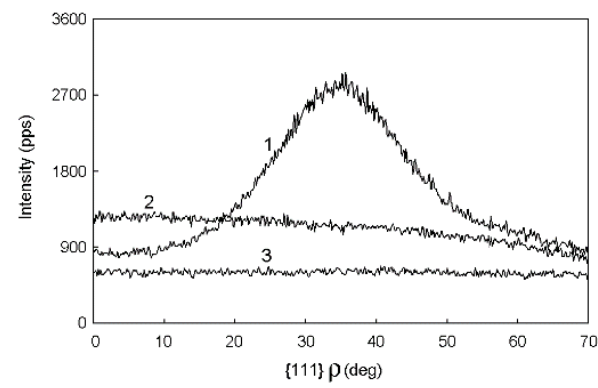

(e)

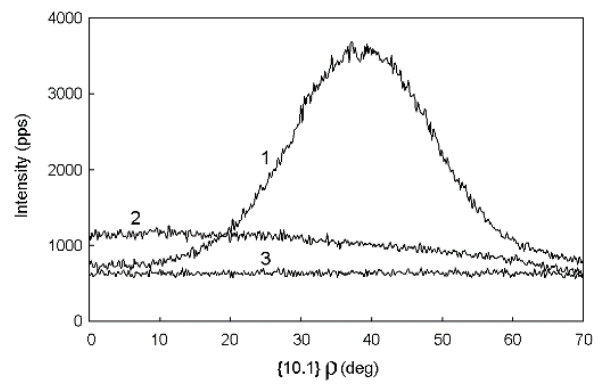

(b)

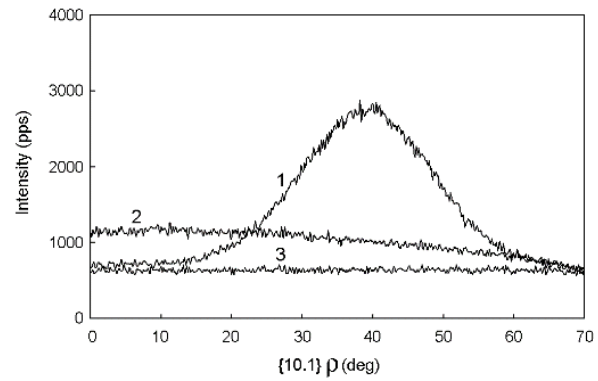

(d)

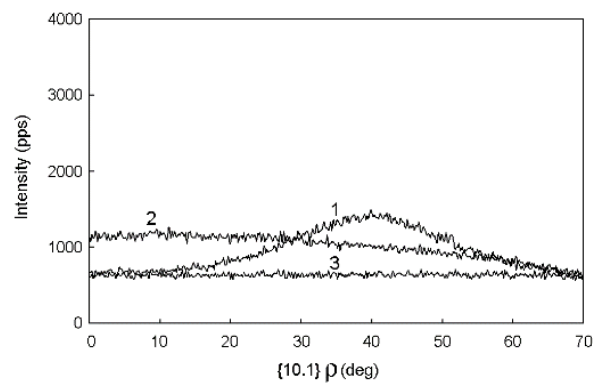

(f)

Fig. 1 Texture-relevant curves of interference by $\{111\}$ for $\beta$-modification (a, c, e) and $\{10.1\}$ for $\alpha$-modification ( $b, d, f)$ of cobalt electrocoatings, obtained at a current density of 0.5 $(\mathrm{a}, \mathrm{b}) ; 2.5(\mathrm{c}, \mathrm{d})$ and $10.0 \mathrm{~A} / \mathrm{dm}^{2}(\mathrm{e}, \mathrm{f}): 1$ and 2 - texture curves for the coating and reference, respectively, 3 - background curve

Indeed, the presence of the textural maximum of the axial orientation of the grains with the [110] axis at an angle of $35.3^{\circ}$ in pole figure (111) for the $\beta$-modification of a two-phase cobalt sample (Fig. 2, a) in combination with the texture maximum of the axial orientation of the grains with the [11.0] axis at an angle of $40.1^{\circ}$ in (10.1) pole figure for the $\alpha$-modification of the same sample (Fig. 2, b) allowed this conclusion. It should be noted that in the hexagonal close-packed system, where $\alpha$-Co belongs, the [11.0] crystallographic direction and the (11.0) crystallographic plane are mutually perpendicular. 


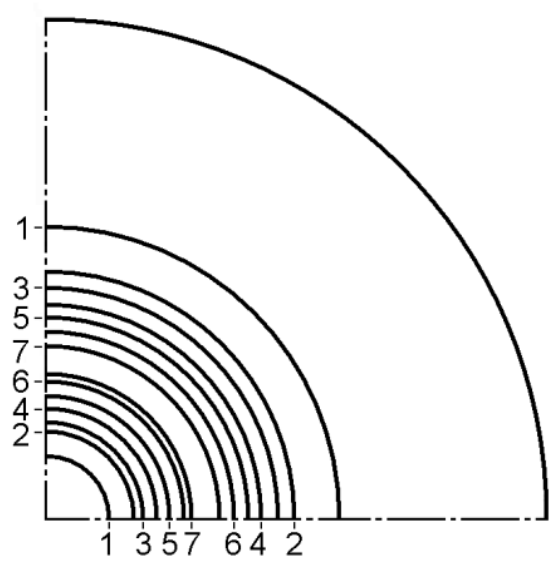

(a)

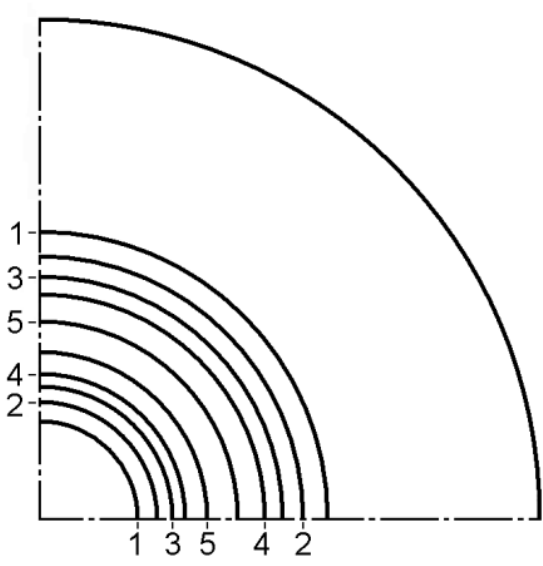

(b)

Fig. 2 Direct pole figures (111) (a) and (10.1) (b) of cobalt electrodeposited at a temperature of $25{ }^{\circ} \mathrm{C}$ and current density of $2.5 \mathrm{~A} / \mathrm{dm}^{2}: 1-7$ - isointensity levels for diffracted X-rays

The results obtained from a qualitative assessment of the preferred grain orientations in various modifications of electrodeposited cobalt are consistent with the data in $[19,20]$, where the axial texture with [110] and [11.0] axes is reported as characteristic of electrodeposited cobalt.

A quantitative assessment of the degree of texture perfection in various modifications of electrodeposited cobalt showed that with an increase in deposition rate (corresponding to a 5 fold increase in the current density: from 0.5 to $2.5 \mathrm{~A} / \mathrm{dm}^{2}$ ), the average scattering angle of the axial grain orientation in $\beta$-Co with the [110] axis decreases significantly (from 12.9 to 10.9 degrees) (Table 1), which indicates a more ordered crystallographic orientation of the grains in the [110] direction. A further fourfold increase in the current density (from 2.5 to $10.0 \mathrm{~A} / \mathrm{dm}^{2}$ ) is also accompanied by the improvement of the $\beta$-Co texture, but to a lesser degree. On the whole, it can be stated that slowing down the process of polymorphic transformation during the electrochemical phase formation of cobalt leads to intense texturing of its metastable $\beta$-modification.

Table 1 Effect of current density on the average scattering angle of axial orientations [110] for $\beta$-modification and [11.0] for $\alpha$-modification of electrodeposited cobalt

\begin{tabular}{|c|c|c|}
\hline \multirow{2}{*}{ Current density $\left[\mathbf{A} / \mathbf{d m}^{2}\right]$} & The average scattering angle of the texture [degrees] \\
\cline { 2 - 3 } & {$[\mathbf{1 1 0}]$} & {$[\mathbf{1 1 . 0}]$} \\
\hline 0.5 & 12.9 & 9.5 \\
\hline 2.5 & 10.9 & 10.9 \\
\hline 10.0 & 10.7 & 13.1 \\
\hline
\end{tabular}

A different nature of the change in the degree of texture perfection with an increase in current density is exhibited by the stable $\alpha$-modification of the electrodeposited cobalt (Table 1). Here, over the entire studied range of current density, the degree of perfection of the axial orientation with the [11.0] axis of the $\alpha$-Co phase grains gradually decreases with increasing the deposition rate of the coatings.

The coatings obtained at a current density of $0.5 \mathrm{~A} / \mathrm{dm}^{2}$ featured intense axial orientation [11.0] of the $\alpha$-Co phase grains with an average scattering angle of 9.5 degrees, while the formation of 
the same phase at a current density of $10.0 \mathrm{~A} / \mathrm{dm}^{2}$ was accompanied by a more significant scattering of texture: the average scattering angle of the axial orientation with the [11.0] axis increased 1.4 times (to 13.1 degrees). Therefore, it can be concluded that slowing down the process of polymorphic transformation during the electrochemical phase formation of cobalt leads to hindering the oriented grain growth in its stable $\alpha$-modification.

\section{Discussion}

The observed change in the degree of texture perfection exhibited by various modifications of cobalt being electrodeposited under slowing down the polymorphic $\beta$-Co $\rightarrow \alpha$-Co transformation can be explained as follows. Since polymorphic cobalt, in a process of its electrochemical phase formation from a liquid state, goes through a metastable $\beta$-Co phase state and crystallizes in the form of a stable $\alpha$-Co modification, slowing down the phase transformation of the metastable modification into a stable one will result in a dual-phase state of the cobalt deposit on the cathode. Slowing down the $\beta$-Co $\rightarrow \alpha$-Co phase transformation caused by an increase in the amount of the metastable $\beta$-Co phase due to an increase in its formation rate, leads to the texturing of the resulting $\beta$-phase. The preferred orientation of the crystal lattices of the grains of the forming $\beta$ phase in the [110] crystallographic direction is primarily due to the tendency of the emerging system of randomly oriented lattices to reduce its internal energy. Therefore, with the slowing down of the process of polymorphic transformation during the electrochemical phase formation of cobalt, an intense texturing of its metastable $\beta$-modification occurs.

Since the $\beta$-Co $\rightarrow \alpha$-Co phase transformation occurs through a shear mechanism, an excessive amount of the $\beta$-phase, continuously forming during electrochemical crystallization, inhibits the phase transition of the metastable $\beta$-phase into a stable $\alpha$-phase. This contributes to slowing down the process of polymorphic transformation, which leads to the formation of a more scattered axial texture with the [11.0] axis in the stable $\alpha$-modification of electrodeposited cobalt.

The above features of texture formation during electrochemical crystallization of polymorphic cobalt were observed experimentally. Indeed, under slowing down the $\beta$-Co $\rightarrow \alpha$-Co phase transition proportionally to an increase in the current density, the preferred orientation of crystal lattices of the cobalt $\beta$-phase occurs in the [110] close-packed direction perpendicular to the coating surface (Table 1). At the same time, a disordering of the crystal lattices of the cobalt $\alpha$ phase is observed in the [11.0] close-packed direction perpendicular to the coating surface (Table 1).

The findings are in conflict with the existing concepts about the electrochemical phase formation in metals and alloys [7-15]. Indeed, it is not possible to explain the observed effect of formation of a metastable $\beta$-modification of cobalt during room temperature electrodeposition, while it is known to exist in the temperature range from 700 to $1768 \mathrm{~K}$; or emergence of a two-phase $\beta$-Co $+\alpha$-Co structure by "incorporating" the ions from the aqueous solution or forming atoms into the crystal lattice of the cobalt being electrodeposited.

\section{Additional Verification Experiments}

If the proposed explanation of the above features of the texture formation in various modifications of electrodeposited cobalt is valid, then the addition into the electrolyte of substances, leading to the disordering of the crystal lattices of the forming grains of various modifications, should be accompanied by the formation of a more scattered texture. At the same time, the revealed features of the texture formation in various modifications of cobalt should generally be maintained. 
To verify the assumption experimentally, the texture of cobalt electrodeposited from the electrolyte of the initial composition with an addition of carbon particles (fraction $<100 \mathrm{~nm}$ ) at a concentration of $1.0 \mathrm{~g} / \mathrm{l}$ was studied. Since carbon nanoparticles entering into the forming clusters of the liquid phase of electrodeposited cobalt can be considered as modifiers, one should expect the formation of not only a more scattered texture, but also a more fine-grained structure of the deposits.

The results of texture analysis (Table 2) of cobalt, electrodeposited in the presence of nanocarbon particles, clearly demonstrate a compliance with the identified texture features of the interrelated formation of cobalt modifications. Thus, while the degree of texturing of the stable $\alpha$-modification of electrodeposited cobalt gradually decreases with an increase in the rate of its formation (Table 2), the perfection of the texture of the metastable $\beta$-modification increases rapidly (Table 2). At the same time, as follows from a comparative analysis of the data in Table $\mathbf{1}$ and Table 2, the obtaining of the coatings from an electrolyte with an addition of nanocarbon particles leads to a significant scattering of the crystallographic texture for both metastable and stable cobalt modifications.

Table 2 Effect of current density on the average scattering angle of axial orientations [110] for $\beta$-modification and [11.0] for $\alpha$-modification of cobalt electrodeposited in the presence of carbon nanoparticles

\begin{tabular}{|c|c|c|}
\hline \multirow{2}{*}{ Current density $\left[\mathbf{A} / \mathbf{d m}^{2}\right]$} & The average scattering angle of the texture [degrees] \\
\cline { 2 - 3 } & {$[\mathbf{1 1 0}]$} & {$[\mathbf{1 1 . 0}]$} \\
\hline 0.5 & 15.8 & 11.2 \\
\hline 2.5 & 12.4 & 12.7 \\
\hline 10.0 & 11.9 & 13.8 \\
\hline
\end{tabular}

A comparison of the surface morphology of the cobalt deposits obtained from electrolytes of different compositions (Fig. 3) indicates the finer grain aggregates of cobalt electrodeposited in the presence of carbon nanoparticles.
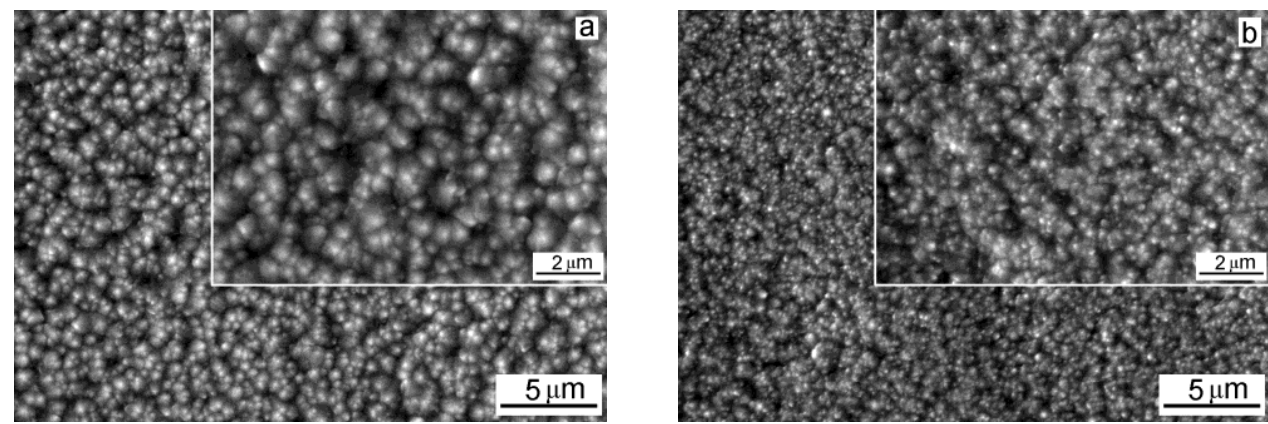

Fig. 3 SEM images of cobalt deposits obtained at a temperature of $25^{\circ} \mathrm{C}$ and a current density of $2.5 \mathrm{~A} / \mathrm{dm}^{2}$ from an electrolyte without additives (a) and with the addition of carbon nanoparticles at a concentration of $1 \mathrm{~g} / \mathrm{l}(\mathrm{b})$

The above results confirm the validity of explaining the observed effects based on the concept of electrochemical phase formation in metals and alloys through a supercooled liquid state stage. Hence, another proof of the existence of the phenomenon of electrochemical phase formation in metals and alloys through a supercooled liquid state stage is an intensive formation of the 
metastable modification texture and simultaneous hindering of the development of the stable modification texture under the conditions of slowing down the polymorphic transformation in the metal being electrodeposited.

\section{Conclusions}

1. A hypothesis has been put forward to consider the crystallization of a polymorphic metal, being electrodeposited from the supercooled liquid state, as its sequential going through metastable modifications and the formation of a modification that is stable under the given deposition conditions as the last stage of phase formation.

2. An idea has been proposed, according to which the slowing down of the process of polymorphic transformation in a metal being electrodeposited should be accompanied by an intensive formation of the texture of the metastable modification and suppression of the texture development of the stable modification.

3. The results of the texture analysis of electrodeposited cobalt as a model metal showed that under slowing down the polymorphic transformation process in a metal being electrodeposited, the texture formation is intensified in the metastable modification, but suppressed in the stable modification. The finding is another proof of the existence of the phenomenon of electrochemical phase formation in metals and alloys through a supercooled liquid state stage.

\section{References}

[1] O. B. Girin: Phenomenon of Precipitation of Metal Being Electrodeposited, Occurring via Formation of an Undercooled Liquid Metal Phase and its Subsequent Solidification. Part 1. Experimental Detection and Theoretical Grounding. In: Materials Development and Processing, edited by J.V. Wood, L. Schultz, D.M. Herlach, WILEY-VCH Verlag Gmbh, Weinheim, Vol. 8, 2000, p. 183-188, http://dx.doi.org/10.1002/3527607277.ch30

[2] O. B. Girin: Phenomenon of Precipitation of Metal Being Electrodeposited, Occurring via Formation of an Undercooled Liquid Metal Phase and its Subsequent Solidification. Part 2. Experimental Verification. In: Materials Development and Processing, edited by J.V. Wood, L. Schultz, D.M. Herlach, WILEY-VCH Verlag Gmbh, Weinheim, Vol. 8, 2000, p. 189-194, http://dx.doi.org/10.1002/3527607277.ch31

[3] O. B. Girin: Journal of Chemical Technology and Metallurgy, Vol. 54, 2019, No. 2, p. 391 396

[4] O. B. Girin: Surface Engineering and Applied Electrochemistry, Vol. 53, 2017, No. 2, p. 137143, http://dx.doi.org/10.3103/S1068375517020041

[5] O. B. Girin: Surface Engineering and Applied Electrochemistry, Vol. 53, 2017, No. 3, p. 233239, http://dx.doi.org/10.3103/S1068375517030048

[6] O. B. Girin: Surface Engineering and Applied Electrochemistry, Vol. 53, 2017, No. 4, p. 339344, http://dx.doi.org/10.3103/S1068375517040056

[7] A. Milchev: Electrocrystallization. Fundamentals of nucleation and growth, Springer US, New York, 2002, http://dx.doi.org/10.1007/b113784

[8] M. Paunovic M., M. Schlesinger: Fundamentals of electrochemical deposition, second ed., WILEY-INTERSCIENCE, Hoboken, 2006, http://dx.doi.org/10.1002/aic.11636

[9] E. B. Budevski, G. T. Staikov, W. J. Lorenz: Electrochemical phase formation and growth: an introduction to the initial stages of metal deposition, WILEY-VCH, Weinheim, 2008, http://dx.doi.org/10.1002/9783527614936 
[10]W. Plieth: Electrochemistry for materials science, Elsevier B. V., Amsterdam, 2008, http://dx.doi.org/10.1016/B978-0-444-52792-9.X5001-5

[11] Yu. D. Gamburg, G. Zangari: Theory and practice of metal electrodeposition, Springer Science, New York, 2011, http://dx.doi.org/10.1007/978-1-4419-9669-5

[12] A. Milchev: ChemTexts, Vol. 2, 2016, No. 4, p. 1-9, http://dx.doi.org/10.1007/s40828-0150021-1

[13]G. Staikov: Nanoscale, Vol. 8, 2016, No. 29, p. 13880-13892, http://dx.doi.org/10.1039/C6NR01547F

[14] T. Mehmood et al.: International Journal of Electrochemical Science, Vol. 12, 2017, p. 12031215, http://dx.doi.org/10.20964/2017.02.45

[15] S. R. Brankovic: Electrochemical Society Interface, Vol. 27, 2018, No. 2, p. 57-63, http://dx.doi.org/10.1149/2.F05182if

[16]D. A. Porter, K. E. Easterling, M. Y. Sherif: Phase transformations in metals and alloys, third ed., CRC Press, Boca Raton, 2009, http://dx.doi.org/10.1201/9781439883570

[17] M. J. Q. Hernandez, J. A. Pero-Sanz, L. F. Verdeja: Solidification and solid-state transformations of metals and alloys, first ed., Elsevier B. V., Amsterdam, 2017

[18]L. Mentar et al.: Transactions of the IMF, Vol. 89, 2011, No. 3, p. 143-150, http://dx.doi.org/10.1179/174591911X13013911711888

[19] S. Armyanov: Electrochimica Acta, Vol. 45, 2000, No. 20, p. 3323-3335, http://dx.doi.org/10.1016/S0013-4686(00)00408-4

[20] A. Vicenzo, P. L. Cavallotti: Electrochimica Acta, Vol. 49, 2004, No. 24, p. 4079-4089, http://dx.doi.org/10.1016/j.electacta.2004.04.001 